\title{
Amplification-Free Identification and Determination of Nucleic Acids by Surface Plasmon Resonance and Surface-Enhanced Raman Spectroscopy
}

\author{
E. K. Pisarev ${ }^{a, ~ *, ~ O . ~ O . ~ K a p i t a n o v a ~}{ }^{b}$ I. A. Vesolova ${ }^{b}$, \\ and M. I. Zvereva ${ }^{b}$ (ORCID: 0000-0002-7432-1574) \\ ${ }^{a}$ Department of Bioengineering and Bioinformatics, Moscow State University, Moscow, 119991 Russia \\ ${ }^{b}$ Department of Chemistry, Moscow State University, Moscow, 119991 Russia \\ *e-mail: e.pisarev@fbb.msu.ru \\ Received May 18, 2021; revised May 20, 2021; accepted May 28, 2021
}

\begin{abstract}
This review discusses contemporary approaches to designing sensory systems for the identification and determination of nucleic acids (NAs) without amplifying target molecules. Here we summarize the data about methods based on surface plasmon resonance and surface-enhanced Raman spectroscopy, as well as their possibilities, limitations, and prospects for further development.
\end{abstract}

Keywords: nucleic acids, analysis without amplification, SPR, SERS, detection of viral and tumorous nucleic acids DOI: $10.3103 / \mathrm{S} 0027131421060079$

In recent decades, there is a high demand in the development of methods for the detection of infectious and genetic diseases based on the identification and determination of nucleic acids (NAs). The application of NAs provides rapidity, low invasiveness, unique specificity, and high sensitivity. Among the approaches used for the identification and determination of NAs for biomedical application, the most popular methods are those based on the polymerase chain reaction (PCR) that allow one to obtain through cyclic enzymatic amplification a great number of fragment copies of the target NA molecule limited by the oligonucleotide sequences required for the in vitro amplification of NA with DNA polymerase [1].

Multiple in vitro enzymatic replication of the original NA in the studied sample of a biological material allows one to detect single molecules (Fig. 1a). At the same time, because of enzymatic replication, errors can be introduced to the resulting fragments upon PCR as single nucleotide substitutions in NA sequences (Fig. 1b). This imposes constraints on the use of PCR methods for the determination of a low-abundance mutant allele fraction of NA, i.e., in the cases when it is necessary to determine a fragment with a point mutation at a large number of wild-type fragments. If the rate of the determined mutation is lower than the error of enzyme operation, the use of the PCR method results in invalid data [2] (Fig. 1c).

Because of the need to analyze circulating cell-free NAs for fetal diagnostics and diagnostics of cancer and viral diseases, the question acutely arises as to whether methods for the identification and determination of NAs in biological liquids are reliable, which requires the development of new approaches allowing one to take into account the error of enzymatic replication. Among the solutions to this issue are droplet digital PCRs operating with the absolute number of NAs [3] and targeted deep Next-generation sequencing using molecular identifiers to estimate the enzymatic replication error at each position [3]. The NA fragments obtained in PCR are detected using different physicochemical methods, for example, electrochemical methods [4-6], fluorescence spectroscopy [7, 8], infrared absorption spectroscopy [9-11], and others [12]. It should be noted that due to the presence of enzyme inhibitors in biological liquids, PCR-based methods are not applicable for the direct detection of NAs in them without the preliminary isolation of the NAs. In the case of the analysis of microRNA, all the above-mentioned disadvantages are escalated by an additional stage, reverse transcription PCR (RT-PCR, transcription of RNA chains into DNA), which makes the analysis more difficult or expensive. Taking into account that RNA-dependent DNA polymerases possess extremely low toxicity, it is obvious that PCR-and RT-PCR-based methods cannot provide reliable data for research purposes requiring the detection of point mutations of genes or their transcripts [13].

Among the promising ways to overcome the current limitations of diagnostic sensor systems is the development of direct methods (without PCR amplification) for the identification and determination of NAs at the level 
PCR amplification of the fragment of a unique NA (the NA contains a target sequence with specified length)
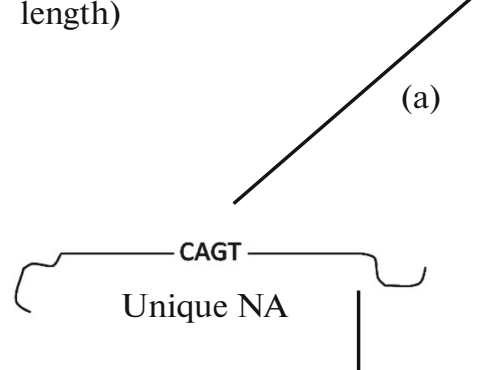

(d)
Random mutagenesis in the PCR product

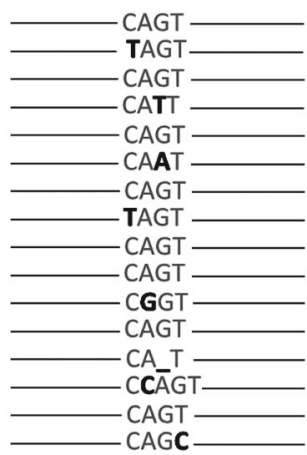

Emergence of a signal from nontarget sequences results in the detection of molecules, which are really absent in the sample

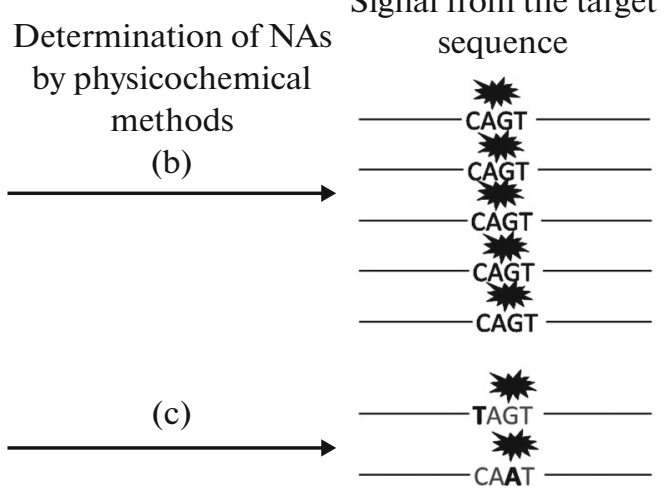

Determination of NA thods

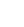

Direct determination by the physicochemical 
the refractive index of the metal film. This change is recorded by a detector $[19,20]$.

Typically, the specific SPR determination of target molecules of the above-mentioned analytes is based on their hybridization resulting in the binding of the NA analyte to the sensor surface with an immobilized oligonucleotide comlementary to the studied sequence, which is called the DNA probe. Such DNA probes can contain or be free of additional indicator molecules (labels) for the implementation of the SPR. In the case of the former, the signal is recorded upon a change in the space position of the label relative to the plasmon surface formed by nanoparticles as a result of conformational changes of the probe and, in the latter case, the signal is recorded upon DNA-DNA (probe-analyte) or DNA-DNA-protein (probeanalyte-DNA-binding protein) complex formation. There are different versions of immobilization of DNA probes on the metal surface: the most popular version is covalent binding through the thiol group $(-\mathrm{SH})$, while modification of the surface with streptavidin and NAs with either biotin or an aptamer adapter sequence is applied more rarely [20]. Substrates are usually created using nanostructured gold or silver [21] and more rarely other transition metals and materials based on them [22]. The use of gold is preferred due to its lower corrosion, lower toxicity for biological objects, and stronger $\mathrm{Au}-\mathrm{S}$ bond $\left(418 \mathrm{~kJ} \mathrm{~mol}^{-1}\right.$ [23]) compared to $\mathrm{Ag}-\mathrm{S}$ (217 $\mathrm{kJ} \mathrm{mol}^{-1}$, according to https://www.webelements. com/silver/compound_properties.html). A significant advantage of the SPR method consists in its high sensitivity due to multiple signal amplification (by factors of $10^{4}$ to $10^{10}$ ) and the absence of the need to use special chemical modifications in some versions.

The SPR method was used also without combination with probe-analyte hybridization to design a system version for the determination of the NA sequence by the type of Next-generation sequenator (NGS) and the development of an SPR biosensor based on it for the determination of the primary structure of the DNA by synthesis. In this version, the system composed of a gold plate with the surface modified with biotinylated dextran was used. The model NA analyte was synthetic oligonucleotides crosslinked with streptavidin. dNTPs of each type were supplied sequentially and removed into a chip flow cell and the addition of a nucleotide was recorded by the change in the refractive index caused by the shift of DNA polymerase in its complex with DNA after the addition of a nucleotide [24]. SPR sensors can provide relatively high sensitivity of about $2000-4000 \mathrm{~nm}$ of the spectral resonance shift per refractive index unity (RIU), but cannot meet modern trends in the development of biochemical analysis, i.e, new hierarchic structures, resolution beyond the diffraction limit, and selectivity by the size of biocomplexes. The surface structuring with arrays of nanoparticles and localized surface plasmon resonances (LSPRs) related to such 2D organization can become a basis for some promising biosensor nanoarchitectures and provide new functional capability. In combination with a large surface for the immobilization of organic compounds and biological objects provided by the $3 \mathrm{D}$ matrix, the proposed sensor architecture prognosticates a new stage in the development of plasmon sensor technologies. Finally, the possibility to amplify the field at hotspots of the metamaterial matrix makes it possible to perform analysis using one sensor element by surface-enhanced Raman scattering simultaneously with the SPR method. The considerably increased surface area specified by the topography and properties of the material allows one to increase the amount of a biomaterial which could be included in the matrix within the available depth for the probe hybridization [25-27]. In addition, the distance between blocks within a nanostructured sensor can be selected in accordance with the size of biopolymer complexes of interest (NA-protein), which gives access to the additional possibility of realizing the size selectivity, which is important for many tasks, and in particular, for the detection of viruses and DNA-binding proteins. In general, the presence of regular cells with a size suitable for target analytes in the array of blocks for surface assembly (a certain 3D morphology of a sensor used in each work is also the characteristic of nanostructuredness) can favor an increase in the reproducibility and sensitivity of the determination of a different nature of biopolymers due to the improvement in the signal-tonoise ratio [28]. Such an approach was implemented in the version of the SPR [24] and SERS [26].

A comparative study of the literature data [18-28] showed that amplification-free methods for the determination of NAs based on the SPR attract attention of researchers due to the possibility of amplifying a signal by a factor of 10 million; however, in terms of sensitivity, they are still inferior to the PCR-based amplification methods enabling the detection of single NAs molecules in biological objects. This fact favored the development of approaches to the determination of the above-mentioned molecules by surface-enhanced Raman scattering.

\section{SERS SENSORS FOR THE DETERMINATION OF NUCLEIC ACIDS}

The inelastic scattering of radiation on molecules of matter accompanied by a frequency change is referred to as the Raman effect or combination scattering. The Raman spectrum of a molecule carries information on transitions between vibrational sublevels, i.e., vibrations of atoms and atomic groups within the molecule [3]. However, the probability of Raman scattering is low compared to elastic scattering (one photon among $10^{7}$ ); therefore, signal amplification is a topical problem. When nanostructured metal surfaces interact with the incident light, localized surface plasmons are excited to produce a giant electromagnetic field at hotspots. When an analyte enters such 
hotspots, the analytical signal is considerably amplified. The difference between the energies of the incident and scattered lights corresponds to the vibrational energy of analyte molecules [29]. SERS produces an effect of a multifold increase in the Raman scattering intensity of a substance adsorbed on the plasmon surface when the wavelengths of exciting electromagnetic radiation and Raman scattering are close to that at which plasmon resonance is observed. In this method, the surface is composed of gold or silver nanoparticles, since they possess the highest coefficient of signal amplification.

Using SERS, we can record the intrinsic spectra of both NAs (direct SERS spectroscopy) and the reporter molecules cross-linked with the DNA probe (indirect SERS spectroscopy). Since in the former version the signal from NAs is caused by vibrations of the sugar-phosphate backbone and nitrogenous bases and is independent of the order or sequence of nucleotide residues, the SERS signal is recorded regardless of the molecular structure. Despite the fact that nucleotides with identical length but differing by one residue can be identified in principle using synthetic single stranded DNAs [30], it should be noted that, under the conditions of real biological liquids, extracellular NAs are cleaved by nucleases to result in the appearance of NA fragments with a different length (the normal distribution with a peak of about 146 base pairs (bp) for the DNA). The possibility of alternative RNA splicing and the presence of several alleles of one gene can significantly complicate the identification of the target fragment. The presence of foreign components also plays a certain role, for example, in the case when a mutant DNA containing single-nucleotide substitution is determined against the background of a wild-type DNA, which is often present at concentrations by several orders of magnitude higher than that of the mutant form. It is considered that direct SERS spectroscopy cannot be used to design sequence-specific sensors without additional PCR amplification. Such a method was implemented in the determination of high concentrations of specified-size fragments of the NA analyte, which is also achieved during PCR [16].

It was shown in [31] that NA fragments with a length of 20 and 141 residues, rather than 35 residues, containing oligonucleotide substitutions of the KRAS gene can have individual distinct spectra against a great number of wild-type NAs. Also, the fragment of $141 \mathrm{bp}$ was shown to have peaks in the region of 633 and $684 \mathrm{~cm}^{-1}$, corresponding to the $3^{\prime}$-endo and 2'-endo conformations of deoxyribose, which suggests the simultaneous presence of the DNA A and B forms in this fragment. No such phenomenon was observed in the case of 20 and $35 \mathrm{bp}$ fragments. This is likely due to the fact that short NA fragments with a length of 20 and $35 \mathrm{bp}$ fold to a spatially ordered structure with double helix fragments of one shape, while the secondary structure of the fragment with a length of 141 n.rs. is a more complex combination of DNA A and B forms [31].
Although the authors of [31] showed that the proposed method can determine nucleotides differing in composition and size and having an identical composition but with a different order of bases, this method is scarcely applicable for the analysis of real clinical samples due to the heterogeneity of the NA lengths. Noteworthy that the method for the unification of the fragment length of analytes by the enzymatic cleavage by restriction endonucleases was suggested in this studying. However, as in the above-mentioned work [30], the disadvantage of using single stranded DNA for analysis lies in the selective isolation of one chain from the DNA double helix. Summarizing all of these points, it can be concluded that analysis methods based on the direct SERS determination of individual DNA molecules are interesting from the viewpoint of fundamental chemistry; however, the feasibility of their practical application requires further studies.

The analysis methods by indirect SERS spectroscopy are free of the described above disadvantages and are applied with success to design biosensors in the solution of a wide range of biomedical tasks, such as the determination of DNAs of viruses, bacteria, and oncogene point mutations.

The authors of [32] developed the "on-to-off" sensor to determine mutations in the $K R A S$ gene. Since the $K R A S$ gene can contain many mutations, the authors proposed determining the content of wildtype DNAs (WT-KRAS) in the total DNA of $K R A S$. As the SERS-amplifying surface, the so-called nanorods, elongated filamentary nanoparticles consisting of a gold core and a silver shell, were used. Nanoparticles were immobilized on a thiolated specimen glass and conjugated with oligonucleotide containing the SH group at the 3'-end and the SERSactive label at the 5'-end. The appearance of WT-KRAS in the mixture results in a hairpin opening and a drop in the signal intensity. The sensor was tested in two versions: (1) on isolated wild-type $K R A S$ DNAs and KRAS G12V (mutant forms with point mutations results in the substitution of valine for glycine in position 12 of the protein), and on their mixtures with a different component ratio of the wild-type DNAs and mutant forms; and (2) on the total DNA isolated from two human cell lines, one of which contained mutations and the other one that was free of mutation, as well as on their mixtures with a different ratio of the wild-type DNAs and mutant forms. The results of the determination were found to be statistically valid. Among the undoubted advantages of this method are the low limit of detection (50 fM) of the $K R A S$ gene DNA, short analysis time ( $\sim 40 \mathrm{~min})$, and low cost. However, while the authors succeeded in showing the statistical repeatability of the data by three measurements for certain concentration ratios, upon variation in the ratio, the signal in the wild-type DNA samples becomes statistically indistinguishable from that in the samples containing $10 \%$ of the mutant form. This imposes a constraint on the content of the DNA of the 
cancer cells in the sample: it should be sufficiently high (as judged by the available data, at least $50 \%$ ). This significantly makes the application of this sensor difficult to use in the diagnosis of cancer.

In some modifications, the sensor is designed from two probes, one of which is typically soluble and conjugated with a metal nanoparticle (required for signal amplification upon approaching the surface) and the second one is attached to the surface and functions as an anchor. The probes are designed so that the target DNA sequence could bind both probes simultaneously, forming two duplexes. Such an approach is called the "sandwich system" because of the similarity with a sandwich, the assembly of which requires the presence of an analyte.

The classical version of such a system was implemented in [33]. In this work, the authors applied silver nanoparticles onto the surface of poly(styrene-coacrylic acid) microparticles. Then, Ag nanoparticles were covalently attached to SERS-active molecules (one particle per SERS-active compound) and the whole construct was coated with a silica nanosphere. The glass surface was treated with piranha and (3-glycidyloxypropyl)trimethoxysilane (GPTMS), which by means of the epoxide group can bind to both glass (through free $\mathrm{OH}$ groups of glass) and $\mathrm{NH}_{2}$ groups of the heterocyclic bases of the NAs. Individual NAs in the mixture were identified using this sensor. Moreover, all four SERS-active compounds tested in the work have individual peaks. It follows that this system allows one to perform a multiplex analysis, i.e., the simultaneous determination of several targets. However, the limit of detection and specificity of such a system were not determined. Furthermore, the analysis procedure takes more than $12 \mathrm{~h}$ and, therefore, the test is not rapid. In view of this, it can be concluded that this system theoretically can be applied for laboratory studies; however, it cannot be used in clinical diagnostics which requires a high throughput capacity.

Another version of the sandwich system was used in [34]. A soluble probe was conjugated with a cubic nanorattle, the center of which contained a gold core placed in the center of a hollow $\mathrm{Au}-\mathrm{Ag}$ sphere. A SERS-active tag was in the space between the cube faces and the core and, from the outside, the cube was coated with gold in order to prevent the escape of the tag from the space between the gold core and the sphere. The second probe was conjugated with magnetic nanoballs. In the magnetic field, magnetic particles precipitated on glass walls and, if the target analyte was present, the complete system, probe 1-analyteprobe 2, was formed as a result of the hybridization. The Raman signal amplification factor of the system with rattles was by three orders of magnitude higher than in case of usual gold nanoparticles. Such a difference is caused by at least two reasons: (1) accumulation of a SERS-active compound between the gold nanoparticles and the Au-Ag shell; and (2) the several- fold amplification of the electromagnetic field in this space. Taking into account that the amplification of the SERS signal depends on the biquadrate of amplification of the near electromagnetic field, the signal intensity will increase by several orders of magnitude. The authors of [34] successfully used such a system to determine the single nucleotide polymorphisms of the $P$. falciparum pfk_13 gene (the limit of detection was $100 \mathrm{amol})$. An important additional result of the considered work was the temperature effect on the specificity of such a type of biosensors. It was shown on synthetic substrates that a decrease in the temperature reduces the specificity of the wild-type sequence probe to DNA targets of this type. The ratio of the intensity of the wild-type target to the mutant sequence with one mismatch upon duplex formation was $8: 1$ and $2.5: 1$ at 37 and $25^{\circ} \mathrm{C}$, respectively.

In the more advanced version [35], the hybridization with the RNA analyte was carried out in a glass capillary containing several washing buffers. A permanent magnet was placed near the capillary. The capillary was moved so that the magnet could sequentially influence all buffers. Accordingly, the magnetic particles moved together with it, which enabled the elimination of the nonspecific binding. The SERS analysis was carried out in the last buffer. The concept of this sensor was named "Lab-in-a-stick." The sensor of such a type, free of the disadvantage related to longterm washing, was successfully tested on both synthetic samples and the RNA of the malaria parasite; in particular, the limit of detection was determined to be 2 amol (the sample volume was $10 \mu \mathrm{L}$ ). The application of such a method for real clinical blood samples of infected patients gave positive results. However, it should be noted that, upon the analysis of real objects, the signal intensity was found to significantly change upon a change in the incubation time from 3 to $5 \mathrm{~h}$. The signal intensity in uninfected samples decreased and the intensity in the infected samples increased, which increased the difference in the intensities of the infected and uninfected samples; this difference became statistically significant. This is probably due to the slow formation kinetics of the sandwich complex.

A nonstandard approach to the synthesis of nanoparticles was realized in [36]. In this case, one soluble probe was immobilized on a gold nanoparticle with a gold core and a SERS-active compound in a void space with a shell structure. The second probe was conjugated with silver microspheres (Ag-HMS) formed using the Lactococcus lactis subsp. cremoris bacteria. The system was used to study the presence of three microRNAs. Such a multiplex system showed high selectivity and specificity at a sufficiently low limit of detection $(2.72,0.24$, and $2.68 \mathrm{pM}$ for miR-21, miR-122, and miR-223, respectively). Further, a check was performed on the RNA obtained from HepG 2 cells with the double independent control of the amount using quantitative PCR. The developed sensor is multiple for all three RNA targets. The response value 
varied from 90.24 to $92.69 \%$. The results obtained by two methods were closely correlated to each other, although the confidence interval was lower for the quantitative PCR.

The work [25] describes the application of another version of such a type of systems with two metal surfaces. The first surface was formed using a matrix made of periodically recurring gold triangles, which were obtained using DNA nanotechnologies (the surface of such a type was found to be more efficient than metal film) where the DNA probe was immobilized. The second DNA probe was conjugated with a nanoparticle composed of a silver core, an interlayer of a nonfluorescent SERS-active compound, i.e., a malachite green isothiocyanate dye (MGITC), and an outer silica shell. The shell fulfilled three functions: retention of the reporter molecule within the particle sphere, an increase in solubility, and binding of the DNA probe. The probes were selected so that they were complementary to two segments of the HBV NA. Thus, the complete system was assembled in the presence of the target NA. Among advantages of this sensor are the low limit of detection (50 aM) and 100\% specificity (the HBV NA sequence with one unpaired base did not hybridize with the probes). The low limit of detection results from the gold matrix and silver nanoparticles are in close proximity to each other. In this case, the local electromagnetic fields created by plasmons conjugate, which leads to a considerable increase in the signal and a decrease in the detection limit.

One of the SERS analysis versions consists of the application of a molecular sentinel-type sensor. In the classical method, a molecular sentinel DNA probe forms a hairpin-like structure, one end of which is attached to a metal nanoparticle and the other one is modified with a SERS-active label. Upon binding to an analyte, the hairpin opens and the label moves too far away from the surface, as a result of which it does not interact with the near electromagnetic field of plasmons and the signal intensity drops [37] (on-tooff system). This principle was successfully applied to design DNA sensors for targets such as HIV-1, breast cancer, respiratory viral infections, and RNA biosensors (for example, for the marker of the highly infectious avian influenza virus strain). We note that by the immobilization of the probe onto the surface consisting of nanoporous gold discs, the authors of [37] succeeded in determining single molecules and, by the example of the IFI27 and IFI44L protein transcripts, the possibility of the simultaneous detection of several targets was shown [38], which resulted in the design of DNA chips for medical diagnostics.

This approach evolved into the design of an inverse molecular sentinel system. In this version, the surfaceattached probe is initially hybridized with another DNA strand. Upon interaction with an analyte, the probe in duplex is replaced due to the formation of a more stable helix between the unattached DNA and RNA that leads to the formation of a DNA/RNA heteroduplex with the analyte. As a result, the double stranded DNA on the sensor surface dissociates and the probes fold to a hairpin, which results in the signal's appearance. This approach was implemented in the development of a DNA chip to the Dengue virus sequences (DENV4) (the limit of detection was $6 \mathrm{aM}$, but real samples were not tested) [40] and in the development of a sensor for the detection of miR-21 and miR-34a, which play an important role in the development of breast cancer and serve as promising targets for diagnosis [39]. The developed system allows us to quantitatively compare the content of miR-21 in the Au565 and SUM149 cell lines, which agrees with the data of the quantitative reverse transcription PCR, and also possesses multiplicity (the SERS-active tags $\mathrm{Cy} 5$ and Cy5.5 were used). The multiplicity was shown on both synthetic RNAs and RNAs from the MCF-7 cells. Although the limit of detection was not determined, the measurements were carried out at a concentration of 5 to $10 \mathrm{pM}$ (the sample volume was $10 \mu \mathrm{L}$ ). This means that the signal is detected reliably when the content of RNA in the sample is about a tenth or hundredth of a femtomol per liter.

The authors of [41] developed a sensor combining SERS determination coupled with ligation (enzymecatalyzed covalent binding of adapter sequence). In this version, three types of oligonucleotides were used: the first one (a common oligonucleotide) was conjugated with a metal nanoparticle, while the second (wild type) and third one (mutant with point nucleotide substitution at the end) were conjugated with two different SERS-active labels. The probes were designed so that the common probe and one of the probes with a SERS-active labels were hybridized with the DNA of the analyte in a sequential manner. The measurements were performed at amounts of the DNA matrix and oligonucleotides equal to 20 and 100 pmol, respectively. The limit of detection was not determined. However, the considered method is interesting in that the signal and concentration of the sample are interrelated by direct proportion, which increases the accuracy of the method [41].

Despite the many attempts to apply the SERS method for the in vivo analysis of the targets, the first successful test of such a biosensor in the inverse molecular sentinel version using NAs on animals was described recently [42]. The developed system has some advantages: (1) a high level of stability in the skin (the study was performed on postoperative slices), which is in some measure caused by the fact that the system operated in the agar-agar-based matrix; and (2) the presence of the intrinsic peak of the nanorattle being dependent only on its concentration. Thus, the system contemplates the presence of the internal standard, the intensity and position of which remain unchanged upon opening/closing of the hairpin of the Cy7-tagged probe. The sensor in 5\% gel was intracuta- 
neously administered to Yorkshire pigs and the target DNA was added after $10 \mathrm{~min}$. The spectra were recorded at $\lambda_{\mathrm{ex}}=785 \mathrm{~nm}$ prior to and after the addition of DNA. Taking into account the calibration (peak at $\left.1288 \mathrm{~cm}^{-1}\right)$, the intensity of the main peak $\left(506 \mathrm{~cm}^{-1}\right)$ changed by an order of magnitude. This suggests the applicability of such sensors in vivo.

\section{CONCLUSIONS}

This review of the literature suggests that the stage of the proof of concept for the PCR amplification-free determination of NAs through the use of the physicochemical amplification of a signal based on SPR and SERS has been passed. The limited scope of the data is due to the absence of universal basic principles for the efficient design of such systems. Despite the sporadic current data on the use of SPR and SERS systems for the amplification-free detection of NAs, these trends can be considered promising, since the detection sensitivity of the target analytes in some cases reaches the level of single molecules; first of all, this is related to the SERS sensors. In the authors' opinion, the prospects for further development of this approach to detect NAs consist of (1) the use of replacement of the NA sensor strands with an analyte to result in the appearance of the signal by the inverse molecular sentinel principle [39]; (2) the design of highly structured self-rearranged systems capable of forming surfaces, providing a more efficient signal amplification, in the presence of an analyte [35]; and (3) the need to control both the buffer and temperature conditions [41].

\section{FUNDING}

This study was financially supported by the Russian Foundation for Basic Research, project nos. 20-04-60477 (Amplification-Free Analysis of Viral NAs) and 18-29-08040 (Amplification-free Analysis of Extracellular Cancer DNA).

\section{CONFLICT OF INTEREST}

The authors declare that they have no conflicts of interest.

\section{AUTHOR CONTRIBUTIONS}

Information about the contribution of the authors: I.A. Veselova and M.E. Zvereva participated in designing the review concept and the analysis of the data. E.P. Pisarev and O.O. Kapitonova looked for and collected the published data. All the authors participated in writing the manuscript, preparing this review, and discussing the results. All the authors contributed equally.

\section{REFERENCES}

1. Dunbar, S. and Das, S., J. Clin. Virol., 2019, vol. 115, p. 18.

2. Filges, S., et al., Sci Rep., 2019, vol. 9, no. 1, p. 3503.
3. Volik, S., et al., Mol. Cancer Res., 2016, vol. 14, no. 10, p. 898.

4. Karaballi, R.A., et al., Phys. Chem. Chem. Phys., 2015, vol. 17, no. 33, p. 21356.

5. Hajian, R., et al., Nat. Biomed. Eng., 2019, vol. 3, no. 6, p. 427.

6. Santhanam, M., Algov, I., and Alfonta, L., Sensors, 2020, vol. 20, no. 16, p. 4648.

7. Bossert, N., et al., Sci. Rep., 2016, vol. 6, no. 1, p. 37897.

8. Jeong, Y., et al., Biosens. Bioelectron., 2018, vol. 111, p. 102.

9. Xu, J.-Y., et al., Chem. Commun., 2012, vol. 48, no. 25, p. 3052.

10. Hui, X., et al., in Proc. 2020 IEEE 33rd Int. Conf. on Micro Electro Mechanical Systems (MEMS), 2020.

11. Neubrech, F., et al., Chem. Rev., 2017, vol. 117, no. 7, p. 5110.

12. Huber, F., et al., Nat. Nanotechnol., 2013, vol. 8, no. 2, p. 125.

13. Tiberio, P., et al., BioMed Res. Int., 2015, vol. 2015, 731479.

14. Tabasi, O. and Falamaki, C., Anal. Methods, 2018, vol. 10, no. 32, p. 3906.

15. Gu, X., et al., Annu. Rev. Anal. Chem., 2018, vol. 11, no. 1, p. 147.

16. Batool, F., et al., Spectrochim. Acta, Part A, 2021, vol. 255, 119722.

17. Yin, G., et al., J. Raman Spectrosc., 2021, vol. 52, no. 5, p. 949.

18. Sotnikov, D.V., et al., Usp. Biol. Khim., 2015, vol. 55, p. 391.

19. Moran, K.L.M., Lemass, D., and O'Kennedy, R., in Handbook of Immunoassay Technologies: Approaches, Performances, and Applications, Vashist, S.K. and Luong, J.H.T., Eds., New York: Academic, 2018, p. 129.

20. Nguyen, H.H., et al., Sensors, 2015, vol. 15, no. 5, p. 10481.

21. Dendisova, M., et al., J. Raman Spectrosc., 2012, vol. 43 , p. 181.

22. Cong, S., et al., Nat. Commun., 2015, vol. 6, no. 1, p. 7800 .

23. Emamzadeh, M. and Pasparakis, G., Sci. Rep., 2021, vol. 11, no. 1, p. 9404.

24. Cetin, A.E., et al., ACS Sensors, 2018, vol. 3, no. 3, p. 561 .

25. Li, M., et al., Anal. Chem., 2013, vol. 85, no. 4, p. 2072.

26. Wang, X., et al., Biosens. Bioelectron., 2020, vol. 156, 112130.

27. Vohra, P., et al., Sci. Rep., 2018, vol. 8, no. 1, p. 11410.

28. Aristov, A.I., et al., Sci. Rep., 2016, vol. 6, no. 1, p. 25380.

29. Fabris, L., Chem. Nanomater., 2016, vol. 2, no. 4, p. 249.

30. Xu, L.-J., et al., J. Am. Chem. Soc., 2015, vol. 137, no. 15, p. 5149 . 
31. Morla-Folch, J., et al., Angew. Chem., Int. Ed., 2017, vol. 56, no. 9, p. 2381.

32. Wu, L., et al., Nanoscale, 2019, vol. 11, no. 16, p. 7781.

33. Li, J.-M., et al., J. Mater. Chem., 2012, vol. 22, p. 12100.

34. Ngo, H.T., et al., Biosens. Bioelectron., 2016, vol. 81, p. 8.

35. Ngo, H.T., et al., Sci. Rep., 2018, vol. 8, no. 1, p. 4075.

36. Zhou, W., et al., Anal. Chem., 2017, vol. 89, no. 11, p. 6120.

37. Ngo, H.T., et al., Anal. Bioanal. Chem., 2016, vol. 406, no. 7, p. 1773.
38. Ngo, H.T., et al., Anal. Bioanal. Chem., 2014, vol. 408, no. 14, p. 3335.

39. Wang, H.-N., et al., J. Phys. Chem. C, 2016, vol. 120, no. 37 , p. 21047.

40. Ngo, H.T., et al., Analyst, 2014, vol. 139, no. 22, p. 5655.

41. Lowe, A.J., et al., Anal. Chem., 2010, vol. 82, no. 13, p. 5810.

42. Wang, H.-N., et al., Nano Res., vol. 11, no. 8, p. 4005.

Translated by K. Utegenov 